\title{
DEVELOPMENT OF HIGH FIDELITY FINITE ELEMENT MODEL OF MOTORCYCLE TELESCOPIC FRONT FORK
}

\author{
Tan, K. S. ${ }^{*, * * * * *, \#}$; Wong, S. V. ${ }^{* * * * * * * * * *} \&$ Megat Ahmad, M. M. H. \\ * Department of Mechanical Engineering, Faculty of Engineering, \\ Universiti Pertahanan Nasional Malaysia, Sg Besi Camp, 57000 Kuala Lumpur, Malaysia \\ ${ }^{* *}$ Department of Mechanical and Manufacturing Engineering, Faculty of Engineering, \\ Universiti Putra Malaysia, 43400 UPM Serdang, Selangor, Malaysia \\ ${ }^{* * *}$ Road Safety Research Centre, Universiti Putra Malaysia, 43400 UPM Serdang, Selangor, Malaysia \\ ${ }^{* * * *}$ Malaysian Institute of Road Safety Research, 43000 Kajang, Selangor, Malaysia \\ E-Mail: keansheng@upnm.edu.my (" Corresponding author)
}

\begin{abstract}
This paper describes the development of a fully deformable finite element model of an upright telescopic motorcycle front fork in LS-DYNA environment. The modelling for each of the key components, overall assemblage, and contact interactions involved were presented. Comprehensive validation was performed by comparing the reaction forces in a quasi-static test performed on a universal testing machine with customised fixture, which was specially designed to impose compressive and bending load simultaneously on the fork unit throughout the process. The behaviour of the fork under such loading conditions was studied and explained. Significant localised deformations observed on the components were also identified and compared. The results from the simulations were found to be well agreed to that of the physical testing, with closely matching profile of the reaction forces and a deviation of $1.4 \%$ based on the work done to deform the fork. Major considerations for establishing the full model were concluded and recommendations were suggested for improvement.

(Received in September 2015, accepted in March 2016. This paper was with the authors 2 months for 2 revisions.)
\end{abstract}

Key Words: Finite Element Modelling, Fully Deformable Model, Motorcycle Fork, Quasi-Static Simulations

\section{INTRODUCTION}

In a frontal collision event, such as a motorcycle crashing into a four-wheeler, the motorcycle frontal components, especially the wheel-tyre assembly, is the part that always makes first and direct contact with the opponent vehicle. While it is the front wheel that first come into contact with the external impacting surface and received the direct load, the front fork on the other hand forms the link that allowed the load to be transferred from the wheel to the frame. It was observed in a full crash test of a motorcycle which crashing onto the side of a passenger car [1] that the wheel deformed as the fork retracted and bent rearward progressively until stopped by the engine block. In another study [2], it was demonstrated that the fork experienced significant permanent bending in a motorcycle to rigid barrier impact test conducted with an impact speed of $32.2 \mathrm{~km} / \mathrm{h}$. In such cases where the fork was shortened substantially besides sustained severe deformation, the geometry of the front end of a motorcycle was significantly altered which would influence the behaviour of the motorcycle and so the rider in the collision. In view of this, in the simulations of motorcycle crashes, actual deformations sustained by the fork should also be accurately captured rather than just represented with a simple force-deflection model.

Undoubtedly, finite element method (FEM) has become the most popular and powerful analytical tool for studying a wide-ranging engineering and physical problems, for it being an analysis of overall behaviours in the design of a simple structure [3], or of complex localised behaviours such as the delamination of composite materials [4]. While various methods and 
the associated algorithms, for example the combined finite-discrete element method (FDEM) presented in [5], are being developed continuously to overcome the limitations of the FEM, increasingly including greater details in modelling complex systems in a quest for higher fidelity and realistic model is also an important trend in the FEM advancement. Full vehicle crash simulations, including passenger car and motorcycle, are one of the representative examples with such progression. However, in spite of a great deal of work that has been done in motorcycle crash simulations since $1970[6,7]$, dedicated modelling works for the fork was generally not seen in literatures. The early simple representation of the fork unit was a rigid link, which was then enhanced by incorporating bending and sliding spring-damper features to represent the stem-triple clamp region and fork tube-slider interaction, respectively [8]. Proper modelling of compression and bending behaviours of the fork was emphasised in [9] but the fork was a multibody (MB) representation. The fork model with a deformable stem root was first seen presented in [10] in the development of a multibody-finite element (MBFE) simulation model, where the nonlinearity in material strength of the root region was given special consideration as it sustained large plastic deformation in collisions. In other MB-FE models [11-14] and also more comprehensive FE models as presented in $[15,16]$ for full motorcycle crash simulations, no special consideration was reported on the modelling aspect of the fork unit. In a more recent development of an exploratory study of applying airbag on motorcycle using simulation, a fork was also simply represented with links [17]. It is thus can be seen that incorporation of the detailed fork model in simulations is generally not gaining much of interest, possibly due to perception that not much can be done on the fork to improve the safety and crashworthiness of motorcycles. Nevertheless, literature survey unveiled that studies of structural behaviour of the fork itself is also relatively limited, which somehow implies that the effects of the structural behaviour of a fork on the overall behaviour of motorcycle-rider systems during crashes is indeed still not well understood. Thus a high fidelity fork model capable of simulating the behaviour of the fork realistically is of utmost important in providing a valuable tool to allow for extensive studies of the subject matter, besides in improving the overall quality of the motorcycle crash simulations.

In the earlier work [18], a detailed fork model has been developed and validated. However, the validation of the fork model was performed against a series of direct frontal impact tests whereby comparisons were made against the kinematics of the striker that imparted the impact load on the fork structure, instead of comparing the response of the fork itself. In view of this, the present work was carried out to further validate the model in a more comprehensive way by comparing reaction forces from a quasi-static test conducted using a universal testing machine, which would impose bending-compressive loads to the fork. Besides, with the aid of the developed fully deformable model, the response of the fork under such load was also studied. The FE modelling aspects were firstly described, and then the physical test setup for the validation was presented together with the equivalent simulation model. These were followed by the results and discussions, along with explanations on the responses of the fork under the test conditions. The simulation was performed using LSDYNA implicit solver [19].

\section{FINITE ELEMENT MODELLING OF THE STEERING-FORK ASSEMBLY}

\subsection{Assemblage of the model}

The fork unit of reference is an upright telescopic type that often fitted on light duty motorcycles with single triple clamp, such as Modenas KRISS 110 [20] (dry mass $100 \mathrm{~kg}$, wheelbase $1245 \mathrm{~mm}$ ). An overall assemblage of such fork is shown in Fig. 1, while the 
disassembled physical fork is presented in Fig. 2. The level of detail in the modelling was determined based on the findings of physical tests [21].

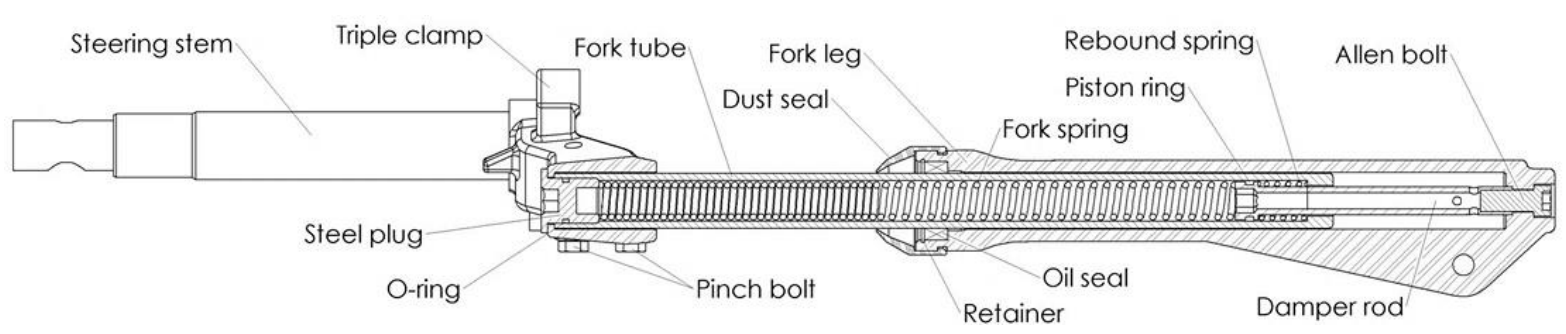

Figure 1: Overall assemblage of a typical telescopic fork.

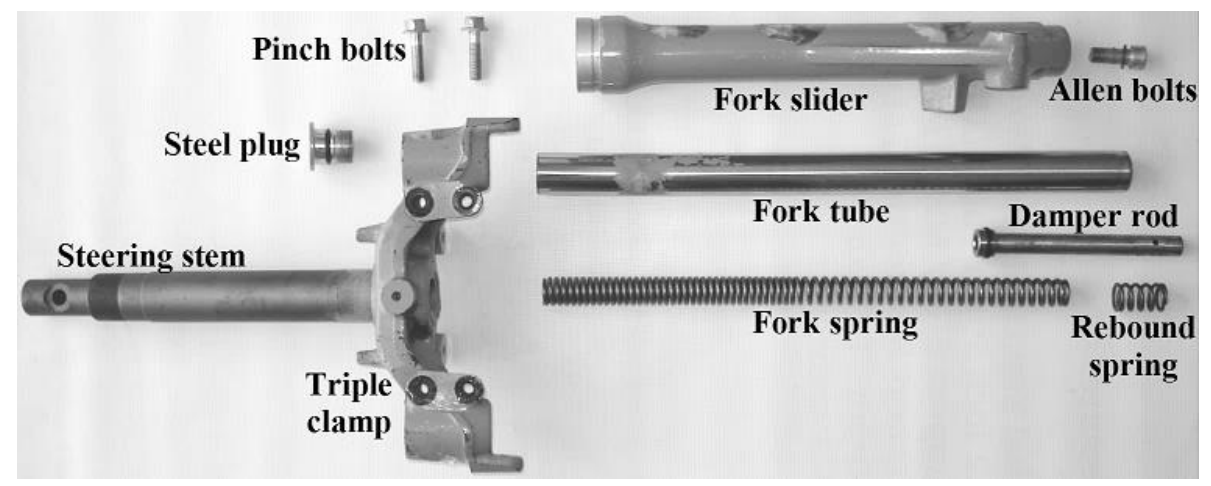

Figure 2: Disassembled fork assembly showing the individual key components.

The triple clamp provides the base where the steering stem and the fork tubes fixed to. At the central cylindrical slot, it holds the stem tightly with the root of the stem welded to the circumferential edge at the bottom side of the slot; at the respective lateral ends, the slightly opened slot that clamps the fork tubes via pinch bolts are just fit enough to accommodate the tube. These joint regions with tight contacts were meshed with relative fine elements to prevent penetration into each other. The stem and triple clamp were modelled respectively with hexahedrons and tetrahedrons. The stem-triple clamp joint region is found to be susceptible to plastic yielding which was observed as an opening between the stem and the slot under a bending load [21]. This represents one of the key deformation characteristics of the fork structure and it is crucial to model explicitly the stem-triple clamp joint region for the model to realistically and accurately simulate the response of the fork. Thus, in the model the stem is not merged to the triple clamp and the weld joint was modelled with TIED_NODE_ TO_SURFACE contact. Other contacts between the steering stem, triple clamp, steel plug and fork tube are treated by AUTOMATIC_SURFACE_TO_SURFACE. Each fork tube is fixed to the rigid steel plug via TIED_SURFACE_TO_SURFACE_OFFSET to represent the thread joint between them. The fork tube, though having wall thickness of $3.5 \mathrm{~mm}$, was modelled with hexahedral elements instead of shells in order to meet the gap requirements imposed by the solver [19]. The resulted gap would be far more than the permissible distance if the fork tube is meshed with shell elements.

In studying the structural response of the fork under external loads, the localised deformation of the thread is insignificant and not of main concern. The inclusion of the pinch bolts in the model would be primarily to simulate the clamping load on the fork tube. Thus, they were thus modelled with a shell-beam system with nodal rigid body representing the thread connections, as in Fig. 3, instead of solid part. Each bolt shank was represented by a Hughes-Liu beam element $[22,23]$ so that the preload in the bolt can be modelled using the keyword INITIAL_STRESS_BEAM. The ISO Standard 15071 [24] was used as the reference to quantify the preload value. 


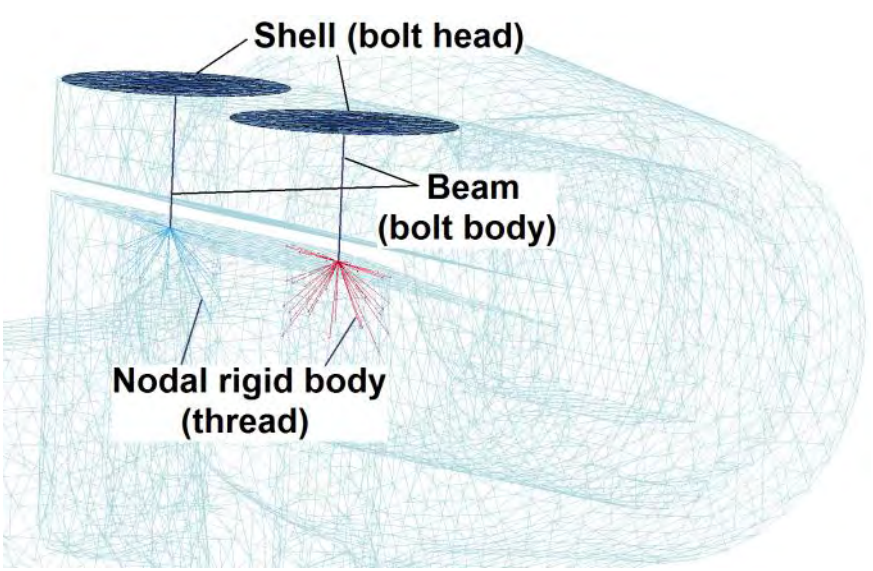

Figure 3: Simplified representation of the individual pinch bolts by a shell-beam-rigid link system.

The average wall thickness of the fork leg is only $5 \mathrm{~mm}$. This, coupled with a tight sliding contact, i.e. the gap of only $0.25 \mathrm{~mm}$ between the corresponding contact surfaces of the fork leg and the fork tube, required that the relatively fine meshes to be modelled for the fork leg. This is crucial not only to prevent undesired initial penetrations but also to capture realistic sliding contact forces during the sliding motions. The sliding contact was modelled with SURFACE_TO_SURFACE_SMOOTH to capture the smooth finishing of the precision tube.

Careful examination on the assembly of the fork revealed that in order to impose correct constraint on the model so to capture the realistic limiting motion, the counter-action of the spring system has to be simulated, thus dictated that the damper rod needs to be incorporated in the model. The counter-action ensures the proper working of the return stroke mechanism, so that the fork tube would not move in subtle motion during the return stroke. This is important for investigating the response of the fork under the quasi-static bendingcompressive load and also to allow for comprehensive validation. The piston of the damper rod provides the supporting base for the rebound and fork springs to be attached to. The damper rod and Allen bolt are attached to the fork leg by TIED_SURFACE_TO_SURFACE. Both the rebound and fork springs are of compression type, which was defined by specifying a non-zero value for the variable CLEARANCE in the keyword SECTION_DISCRETE. The damping elements were not incorporated in the simulation of static loading.

Most of the contacts were of local type in order to improve convergence and in some cases the use of the automatic type of contacts has been found to improve the convergence. The reaction forces that equivalent to the one recorded by the machine in the physical test were obtained via the contact SURFACE_TO_SURFACE between the axle and the upper supporting base.

\subsection{Modelling of materials}

Parts that required detail modelling which necessitates the definition of the material input data up to plastic regions are the steering stem, triple clamp and fork tube, due to their significant deformation sustained in the tests [21]. These data are not available from the manufacturer and usually to be determined by physical tests. It was noted that as the loading rate to be considered was in the quasi-static range and simulations were to be ran using an implicit solver, thus strain rate parameters were not defined in the material models but only required for the cases involving impact [18].

For the stem with the end form of solid round bar, a typical dumbbell shape specimen for tensile test was machined from it and the test was performed to obtain the stress-strain $(\sigma-\varepsilon)$ curve. The data was used as the input and the stem material was defined by Material Type 24, an elasto-plastic material model with an arbitrary stress-strain curve [19]. On the other hand, 
the tensile test was not viable to be performed for the triple clamp and fork tube due to impracticality of acquiring a proper specimen from the corresponding parts. For the triple clamp, its end form is apparently complex while for the fork tube, the relatively small diameter yet thin-walled cylindrical form had also hindered the extraction of any specimen in the desired dumbbell shape.

A simplified inverse approach which based on $[25,26]$ was thus strategised to estimate the material data. In general, the method involved subjecting a whole component of interest to a relatively simple physical test, and the equivalent setup was then numerically simulated with the models incorporated with initial material data obtained from industrial standards or relevant literatures. The simulation results such as time history of reaction forces were then compared against the physical one so that the estimated material input data were to be finetuned progressively until the reasonably matching results are attained. Here, a tensile test by pulling the whole fork tube was apparently not suitable considering that the complication that could result from the need of applying relatively high gripping force to prevent slippage. This could produce localised deformations at the grip region, leading to material yielding at an undesired location and the resulted elongation would then be hard to quantify. An alternative approach adopted in the present study was a typical three-point bending test, as depicted in Fig. 4 showing the arrangement at the end of the test. The displacement rate was set at $1 \mathrm{~mm} / \mathrm{min}$, for a total of $15 \mathrm{~mm}$. Reaction forces versus anvil displacement $(F-d)$, as plotted in Fig. 5, were to be used for comparison.

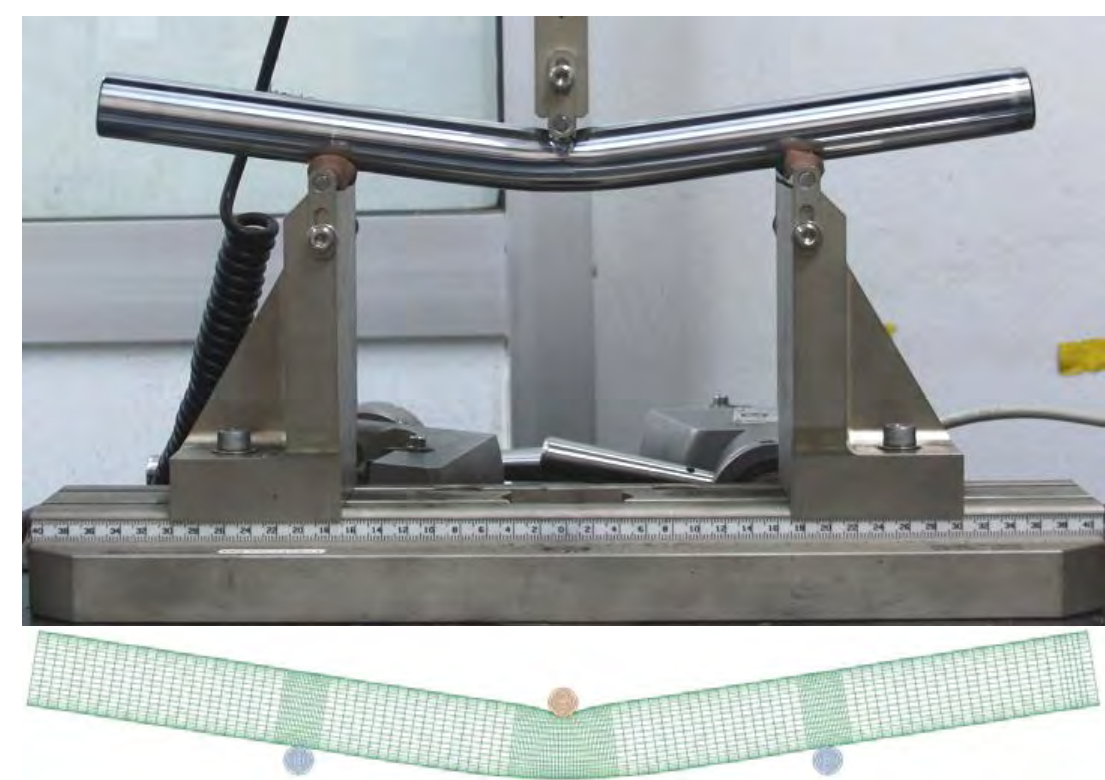

Figure 4: Three point bending test for the fork tube as in the physical deformation of the fork tube in the simulation as compared to the physical one.

The fork tube is typically made of a seamless cold drawn precision steel tube of steel grades St52, St45 and St35. The associated standard is JIS G3445 [27], comparative to EN 10305-1, ASTM A519, and DIN 2391. The corresponding axial direction stress-strain $(\sigma-\varepsilon)$ relationship of the tube material St35 has been modelled with the relations $\sigma=724 \varepsilon^{0.12}$ [28], i.e. Hollomon's power law model $\sigma=K \varepsilon^{n}$, of which $K$ the strength index, $n$ the hardening index. However, the simulation of the equivalent setup performed using Material Type 15, i.e. MAT_POWER_LAW yielded significantly unmatchable results as shown in Fig. 5 of the curve indicated by $K=724, \varepsilon=0.12$. It can be seen that the simulated $F$ - $d$ response is significantly deviated from that of the experimental. Further trials with some other values of $K$ and $n$ also showed no tendency of getting closer to the experimental results. 


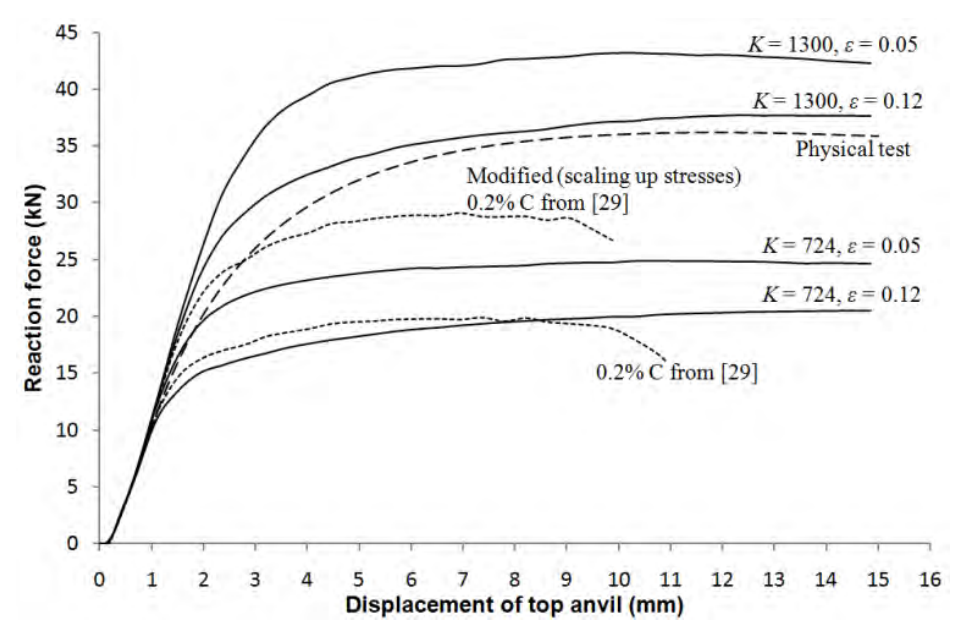

Figure 5: Reaction forces in the respective simulations with different material input data as compared to the physical three-point bending tests.

Further simulations were performed using Material Type 24, with input data of St52 and St45 that based on the material handbook [29]. Again, as shown in Fig. 5, significant deviation in the results was observed. Besides, the simulation also failed to converge at the time corresponding to about $10 \mathrm{~mm}$ of displacement due to the strain bottomed out. Additional trials performed by directly scaling up the stresses of the original data also failed to demonstrate improvement on the results. A comprehensive fine-tuning is thus required, whereby a multi-linear model was adopted to facilitate the point-to-point adjustment of the curve. The approach was started with a bilinear model, which was derived from the aforementioned material data of St52 and St45 in the handbook and progressively refined to a multi-linear model by adding and adjusting the key points on the $\sigma-\varepsilon$ curve, including points in initial yielding region and ultimate tensile strength point. The $F-d$ curve that closely matched the experimental one, which resulted from the simulation using the final fine-tuned material input data, is shown in Fig. 6. The deformation was also closely duplicated in the simulation, as depicted in Fig. 4. These indicate that the material property of the fork tube was reasonably estimated. The estimated material data in terms of true stress-strain values are summarised in Table I.

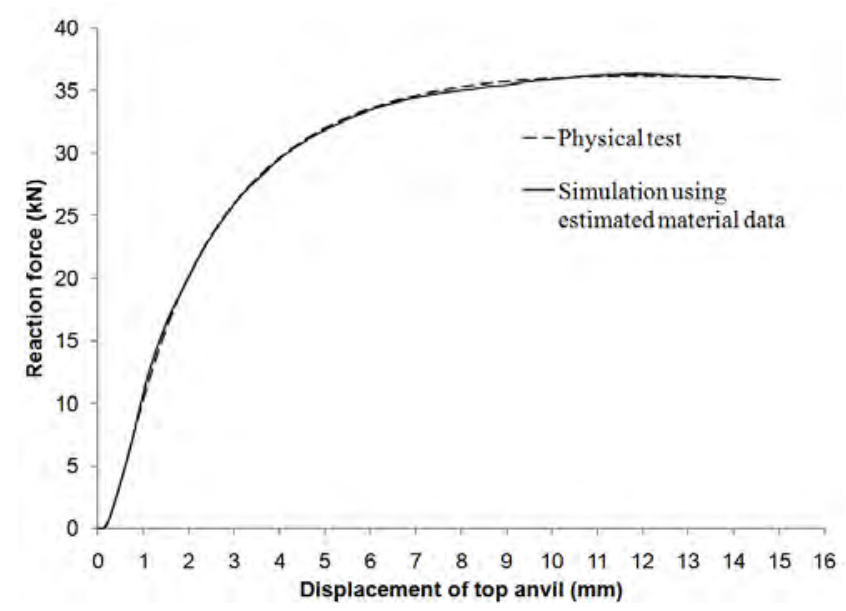

Figure 6: The $F-d$ curve of the simulated three-point bending test that closely matched the physical test.

Table I: Estimated material data for the fork tube.

\begin{tabular}{|l|c|c|c|c|c|c|c|}
\hline True stress (MPa) & 330 & 630 & 760 & 900 & 964 & 975 & 980 \\
\hline Effective plastic strain & 0 & 0.0035 & 0.006 & 0.0182 & 0.035 & 0.041 & 0.190 \\
\hline
\end{tabular}


Obviously, the success of the inverse approach would depend on the accuracy in modelling the geometry of the component. Thus it is not suitable to be applied to the triple clamp because of its complex end form and also difficulty in performing simple component test. However, the fabrication mark resided on the part provides useful information for identifying the fabrication process and so the specific material used in accordance to the standard industrial practice. For the triple clamp, a $5 \mathrm{~mm}$ wide parting line resided on it suggests that the part is made from forging process instead of casting, in which the parting line would be typically 1-2 mm wide. It is known that the material commonly used for the forged triple clamp is carbon steel of grade C20. The corresponding material data in terms of $\sigma-\varepsilon$ curve is available in [29] and thus was used as material input for the triple clamp, defined with Material Type 24.

The fork legs was found sustained no deformation in the physical test [21], but having significant sliding contact with the fork tube. Hence it is sufficient to be modelled with simple elastic material model, with typical properties of aluminium alloy. It is well expected that both the damper rod and Allen bolt would not experience significant deflection, and were represented as rigid parts with typical steel properties.

The fork and rebound springs are made of a dual- and fixed-rate springs, respectively. According to the manufacturer's specification, the preloading of the spring system in an assembled fork is $150.5 \mathrm{~N}$, with total stiffness of $7.8 \mathrm{kN} / \mathrm{m}$ for the first $0.05 \mathrm{~m}$ deflections, which then increases to $18 \mathrm{kN} / \mathrm{m}$ for further deflections. The stiffness of the individual spring is not known and was determined experimentally. The dual rate behaviour of the spring is defined with nonlinear elastic model of Material Type S4 for discrete springs, whereas the constant stiffness with Material Type S1.

\section{RESULTS AND DISCUSSION}

\subsection{Model validation}

The developed FE model was validated against quasi-static tests customised for the fork unit [21]. The tests were performed on a typical universal testing machine as illustrated in Fig. 7, together with the equivalent FE model setup. A customised fixture was used to hold the fork in an inclination of $30^{\circ}$ from the horizontal in its initial position so that both the compressive and bending characteristics of the fork can be studied. The crosshead speed was set at 20 $\mathrm{mm} / \mathrm{min}$. A comprehensive validation was to be performed by comparing the corresponding force-time $(F-t)$ curve. Significant deformations of the post-test fork assembly were also carefully examined for comparison.
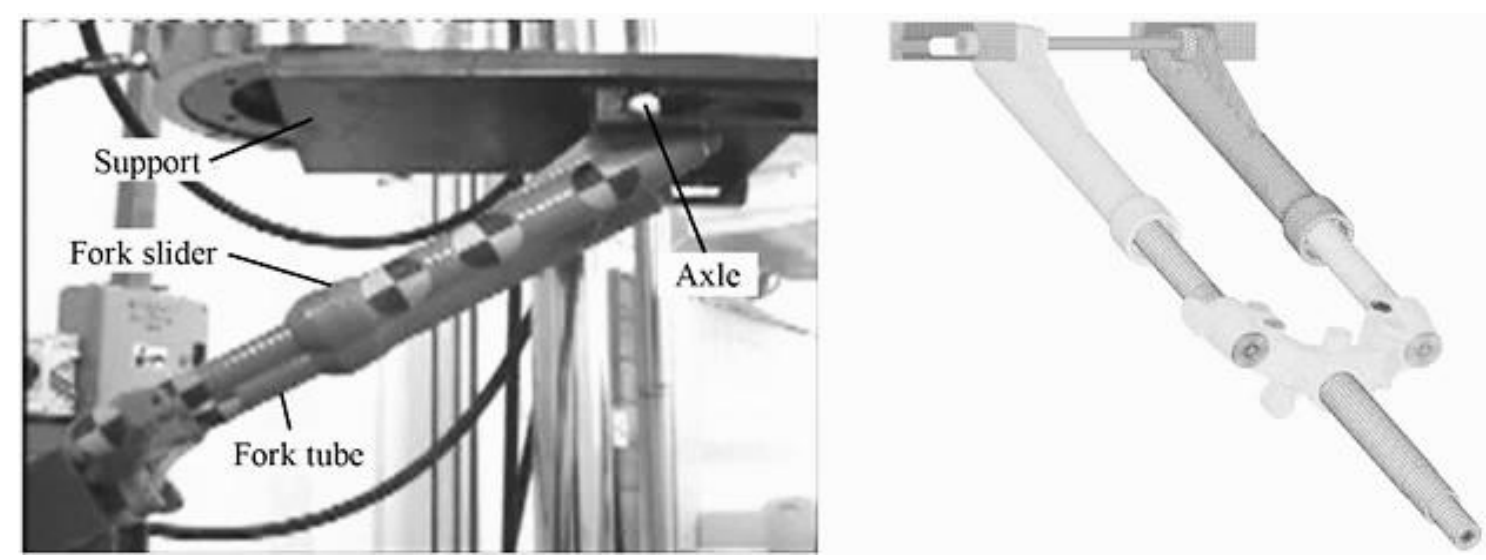

Figure 7: Physical setup of the static test for the fork assembly and the simulation model. 


\subsection{Overall response}

The response curve from the test in terms of vertical reaction force versus time $(F-t)$ is shown in Fig. 8. A replicate test has an approximately the same response curve and is therefore not shown. With inclination at the angle of $30^{\circ}$, it is expected that the fork assembly experienced both the compressive and bending loads the fork assembly simultaneously, with the compression mainly sustained by the springs while the bending by the structural parts. With the elastic bending stiffness of the fork assembly of $280 \mathrm{~N} / \mathrm{mm}$, compared to the only $9 \mathrm{~N} / \mathrm{mm}$ of the stiffness of the spring, the $F-t$ curve is basically dominated by the bending.

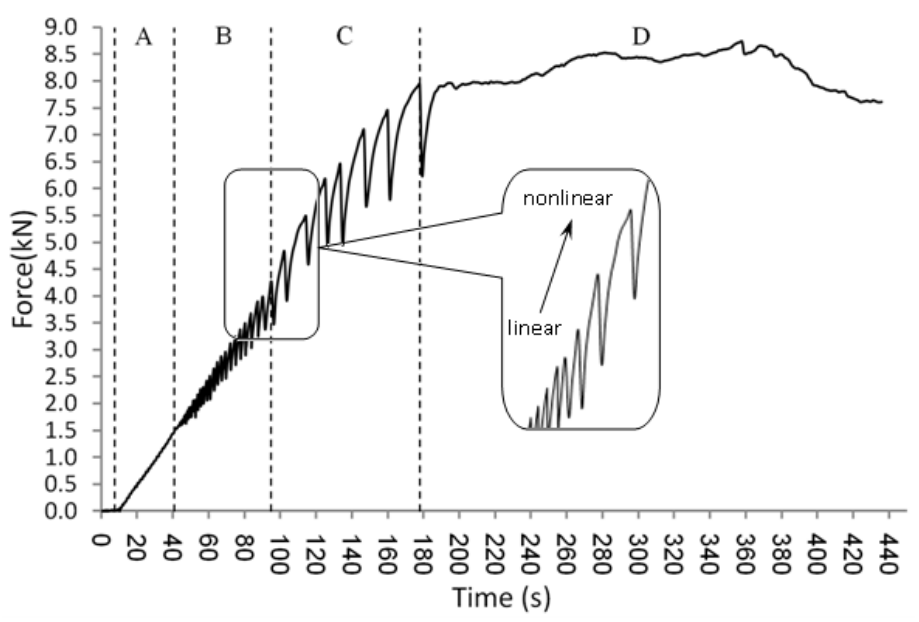

Figure 8: Reaction forces versus the displacement of the cross-head $(F-t)$.

For the test progress in the region A, the fork assembly was getting shortened via a smooth relative motion between the fork tube and the slider. It was observed that in the early stage, the axle has no horizontal movement and was basically acted as a pivot as the crosshead moved up, i.e. the deflection is predominantly the spring compression. The zigzag patterns in the region B was initially speculated as some kind of noise. Until the last few cycles towards the end of the region $\mathrm{C}$ which is having relatively large fluctuations, then only it caught the attention of the associated behaviour that contributed to the fluctuations. It was found that the axle was indeed rolled against the slot as it moved forward for a limited short distance of a few $\mathrm{mm}$, which then immediately followed by a subtle relative retracting motion between the fork leg and the fork tube, also for some short distance, but repeated with gradually increasing magnitude. The rising portions of the zigzags were corresponding to the forward rolling, and the sudden dropping portions to the subtle retractions. Further examination also revealed that when the axle moved forward as the crosshead advanced, there is no further compression on the spring until the retraction, i.e. the compression occurred in discrete stages instead of continuously. It was found that the subtle retraction and the discrete compression of the spring were also noticeable since the early region $B$ at about $55 \mathrm{~s}$.

The configuration of the fork tube and slider with exaggerated misalignment is depicted in Fig. 9. It can be seen as illustrated that as the crosshead advanced, the slider tended to press on the top fillet region of the fork tube, forming a tight contact between the two. The hard pressing and the resulted misalignment, coupled with frictional effects, prevented the fork slider and the tube from sliding against each other instantly in a continuous manner. But with the increasing load the resistance would be finally overcame, followed by the subtle sliding of the fork slider and the spring gets compressed further. The sliding distance was limited due to the higher contact resistance in the following stage, and the process repeated. This limit depends on the overall inclination angle of the fork assembly which progressively reduced as the crosshead advances. The increasing zigzag magnitude suggested that the slider slid further 
in every subsequent cycle. This is explained by the increased misalignment between the fork leg and the fork tube as the overall inclination angle reduces, yielding the higher contact resistance and thus higher force built up which able to compress the spring further once sliding occurred. From the video clips, this was observed as some kind of a sudden retraction of the slider accompanying by a quick backward motion of the axle.

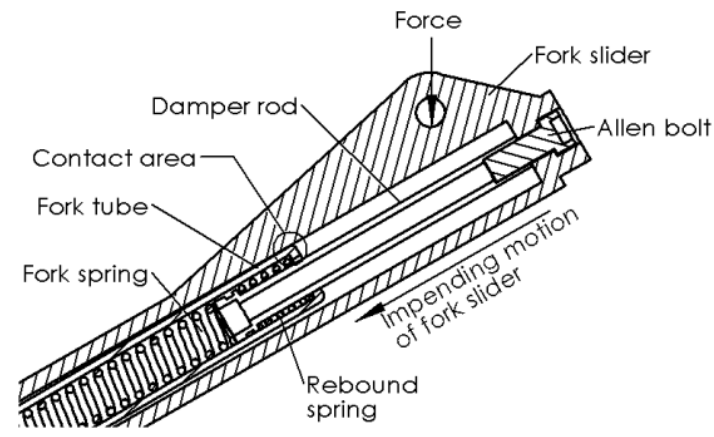

Figure 9: Arrangement of the fork tube and the slider with misalignment exaggerated.

The overall increase of the reaction force was mainly attributed to the increase of overall bending resistance that came from the fork unit. The initiation of the fluctuation signifies that the friction and contact resistance between the fork slider and the fork tube started to become significant. The overall bending resistance was represented by the series of the vertices of the zigzags whereas the nadirs representing the limit of the retraction distance. The forward motion of the axle via rolling against the slot, as mentioned in the previous section, was only noticeable at about $100 \mathrm{~s}$, which indeed was at the beginning of the region C. Besides, at about the same time during the experiment, the opening at the joint of the steering stem-triple clamp was also started to be seen. This suggested the onset of yielding of a certain portion at the joint region which gives the corresponding significant nonlinear portion of the curve. The opening of the joint is illustrated in Fig. 10, with the side view showing the exaggerated deformation. This also explained the zigzags portion in the region $\mathrm{C}$ that apparently rises to higher vertices than those in the region B. With the lower overall inclination, the misalignment between the fork leg and the fork tube tended to increase which would give higher contact resistance and thus resulting in higher force built up before the slider slides. The similar mechanism applies here, where the occurrence of yielding lowers the inclination even further and thus allowed the even higher force to be built up until the slider slides.
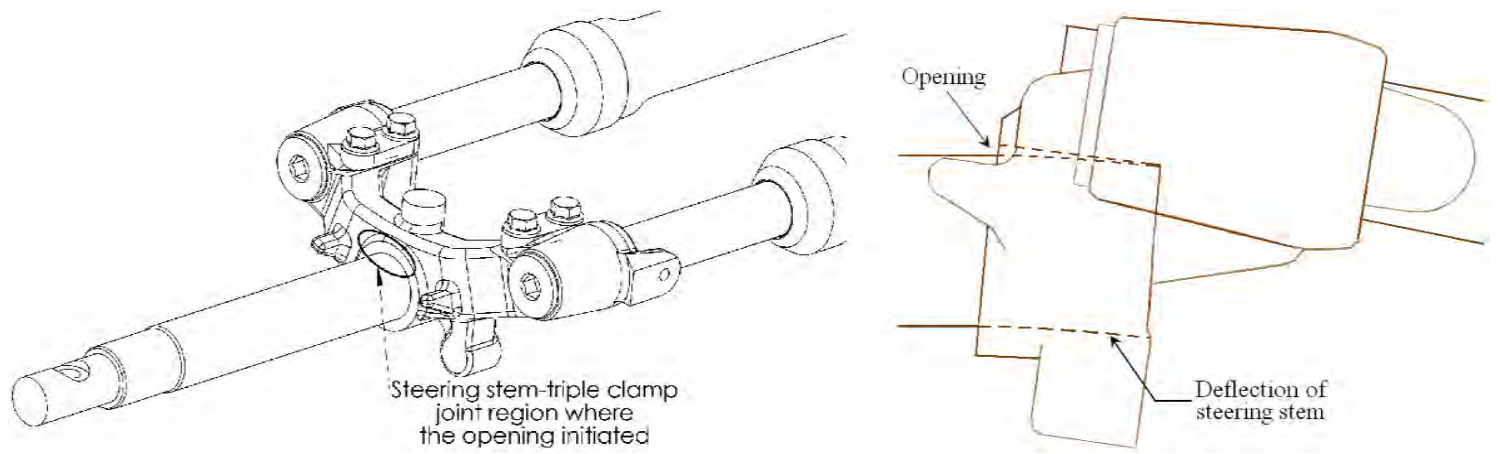

Figure 10: Joint region of steering stem-triple clamp where the opening initiated.

Results in terms of reaction forces from the simulation and physical test are plotted together for comparison, as presented in Fig. 11. It is seen that the response curve of the simulation is generally close to that of test. The respective estimated work done in the Tests 1 and 2 are $710 \mathrm{~J}$ and $730 \mathrm{~J}$, giving an average of $720 \mathrm{~J}$ while it is $710 \mathrm{~J}$ in the simulation, i.e. with a relatively small deviation of only $1.4 \%$. 


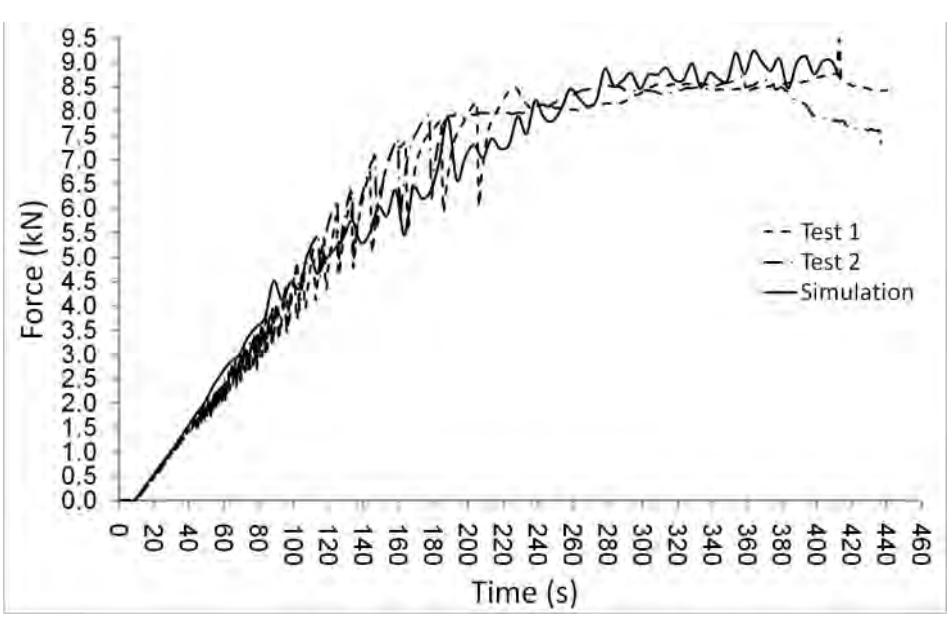

Figure 11: Comparison of the reaction forces between the physical tests and the simulation.

\subsection{Major deformations}

The overall conditions of the post-test fork units are shown in Fig. 12. For the side view, only the fork from the Test 1 is shown as the conditions are basically the same. The only significant deformation in a form of permanent opening was found at the stem-triple clamp joint region, as depicted in Fig. 13. The simulation model shows the comparable results, with the maximum opening of $2.2 \mathrm{~mm}$ compared to the measured value of about $2.5 \mathrm{~mm}$.
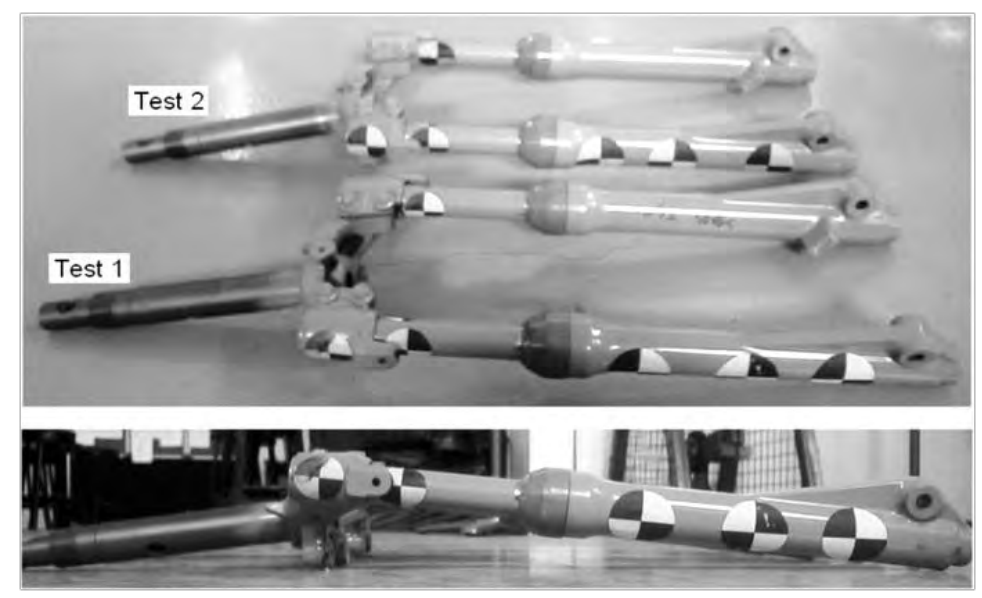

Figure 12: Overall condition of fork unit after testing.
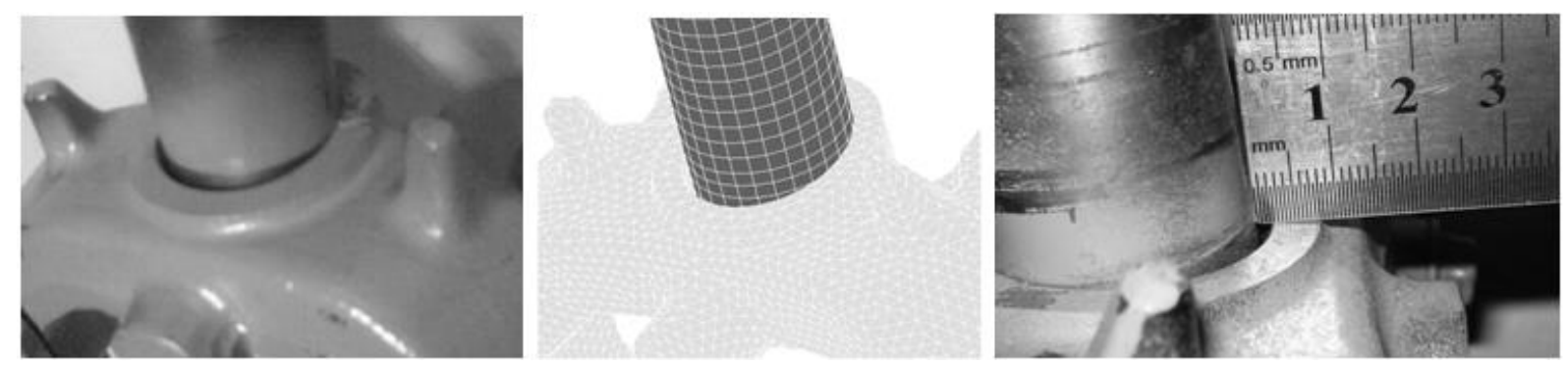

Figure 13: Deformation at the stem-triple clamp joint.

The compression-time $(\delta-t)$ response of the fork spring between the simulation and the experimental was also compared, as presented in Fig. 14. The exact time history data of the compression is not available, which is expected to be a stair-like curve composed of series of steps due to the consistent repeating retractions as discussed earlier. However, with the known starting time and the observed final deflection in the test, an approximated linearised curve 
can thus be produced. The curve starts with $-28 \mathrm{~mm}$ instead of zero due to the precompression in the initial assemblage. The final compression sustained by the spring in the simulation is about the same to that of the physical test. The overall slope of the curve from the simulation is slightly lower and this is explained by different modes of loading that sustained by the springs. In reality, the spring was compressed by series of dynamic forces resulted from the subtle retractions, but in the simulation the compressions occurred in continuous static mode. The deviation is not significant until about $100 \mathrm{~s}$ onwards, which corresponding to the beginning of the nonlinearity in the zigzag pattern of the $F$ - $t$ curve, and then gradually increases with the magnitude of the retraction. The highlighted stepping portions are found to be matched with the retracting motions shown in the simulated animation and this further supports the above explanation.

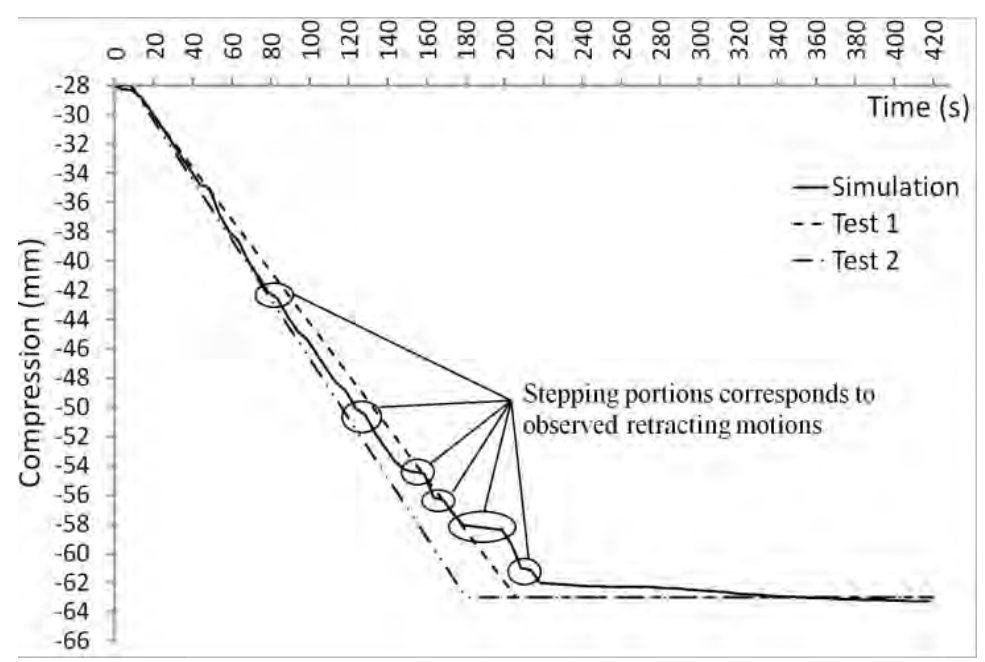

Figure 14: The compression of fork spring in the physical testing and simulation.

\subsection{Discussions}

In general, the simulated response of the fork is consistent to that of the physical test and was able to capture the key structural response of the fork. However, the simulated retracting motion between the fork tube and the slider only occurred intermittently instead of repeating consistently as in reality. Further investigations found that the rolling of the axle against the upper base was not simulated perfectly. This is mainly caused by the meshing quality of the model and the contact algorithm. The sliders sustained no significant deformation in the physical test and thus of no significant concerned. In addition, they have a transition of the circular to ellipse cross sectional shape and some side features such as the rib and the protruding structure. Thus they have been meshed automatically with tetrahedron rather than structured meshed and so the resulted surface is relatively rough. By discretisation, it is also impossible to get a perfect cylindrical surface for the axle slot. These could cause complication to the associated contact treatment as the irregularity prevented the smooth rolling and was worsened with unrealistic penetrations, which are common for penalty based contact algorithm. Some penetrations between the axle and slider slot are depicted in Fig. 15. In simulations, some amount of rolling only observed in the simulation if a zero or an unrealistically low value of coefficient of friction is defined for axle-slider contact. But further examination on the behaviour of the axle shows that it was indeed a spinning motion rather than rolling. To have the simulation results that are closer to the reality, highly refined structured meshes for the contacting surfaces are required. However, the retraction is regarded as trivial to the overall structural behaviour and there is no significant effect to incorporate such feature in the model. 


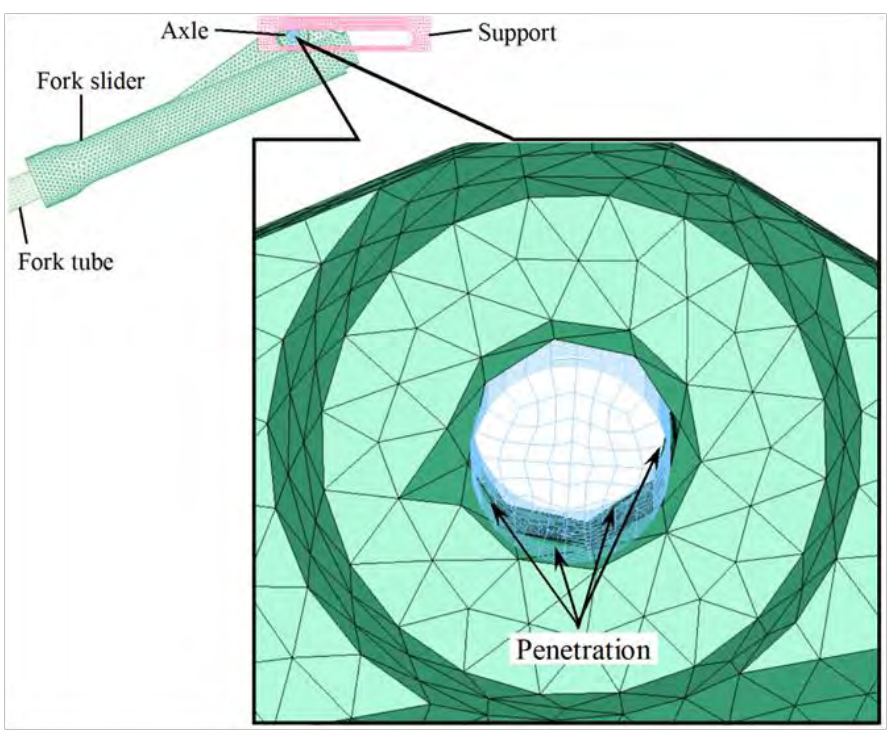

Figure 15: Contact interactions between the axle and the slider slot.

\section{CONCLUSION}

A high fidelity FE model of a motorcycle front fork has been developed and validated. The physical testing method for the validation purpose was specially designed to impose the load in a configuration that reflects the real world scenario so that a more useful and relevant validation was done. This was also to ensure that important behaviours of the fork can be identified and thus be incorporated in the model.

Special concern is required in modelling the connection characteristics between the triple clamp and steering stem, including the geometric fits, material definition and contact treatment, as the significant permanent deformations that occurred in the connection region has crucial effects on the overall structural response of the fork.

Under the coupled compressive-bending load, the response of the fork is sensitive to the frictional properties of the surfaces of the axle, axle slots on the fork leg, and also slots of the external base plate that supports the axle. Though such frictional effect is trivial compared to the overall structural behaviour of the fork, it was useful for extended validation.

The main inadequacy of the model is the lack of exact material definitions for the triple clamp. A practical method shall be adopted to reverse engineer the material properties.

\section{ACKNOWLEDGEMENT}

The authors wish to acknowledge the financial support from the Ministry of Science, Technology and Innovation of Malaysia through Science Fund Allocation, MOSTI No. 03-01-04-SF0549 and the sponsorship of KYB-UMW (Malaysia) Sdn. Bhd. for 12 sets of complete front fork units of MODENAS KRISS 110 motorcycle.

\section{REFERENCES}

[1] Azhar, H.; Mohd Khairudin, R.; Aqbal Hafeez, A.; Mohd Syazwan, S.; Noor Faradila, P.; Mohd Hafzi, M. I.; Zulhaidi, M. J.; Khairil Anwar, A. K. (2014). Motorcycle structural response in simulated vehicular collision, Proceedings of International Crashworthiness Conference 2014, ICR-14-39, 6 pages

[2] Nieboer, J. J.; Wismans, J.; Versmissen, A. C. M.; Van Slagmaat, M. T. P.; Kurawaki, I.; Ohara, N. (1993). Motorcycle crash test modelling, Proceedings of $37^{\text {th }}$ Stapp Car Crash Conference, 273-288, doi: $\underline{10.4271 / 933133}$ 
[3] Moslemi, M.; Khoshravan, M. (2015). Cohesive zone parameters selection for mode-I prediction of interfacial delamination, Strojniski vestnik - Journal of Mechanical Engineering, Vol. 61, No. 9, 507-516, doi:10.5545/sv-jme.2015.2521

[4] Cirkovic, B.; Camagic, I.; Vasic, N.; Burzic, Z.; Folic, B. (2015). Analysis of the supporting structure of composite material tool machine using the finite element method, Technical Gazette, Vol. 22, No. 1, 95-98, doi:10.17559/TV-20130928105016

[5] Uzelac, I.; Smoljanovic, H.; Peros, B. (2015). A new algorithm for dynamic analysis of thin plates in the combined finite-discrete element method, Transactions of FAMENA, Vol. 39, No. 2, 47-54

[6] Knight, R. E.; Peterson, H. C. (1971). Dynamics of motorcycle impact. Volume III, Digital computer simulation of two-dimensional motion of motorcycle and dummy rider, Report No. DRI-2574-3, Denver University, Denver

[7] Knight, R. E.; Peterson, H. C. (1973). Dynamics of motorcycle impact (1971-1973). Volume 3, Computer simulation of three-dimensional motion of motorcycle, Report No. DOT HS-800 908, Denver University, Denver

[8] Chinn, B. P.; Happian-Smith, J.; Macaulay, M. A. (1989). The effect of leg protecting fairings on the overall motion of a motorcycle in a glancing impact, International Journal of Impact Engineering, Vol. 8, No. 3, 265-279, doi:10.1016/0734-743X(89)90006-7

[9] Roggi, M.; Pierini, M. (2007). Optimization methodologies to improve existing protective equipment. Analysis from in-depth data studies and simulated reconstructions, Deliverable D431A, Final Revision, Project No. FP6-PLT-506503, Advanced Protection Systems (APROSYS)

[10] Kanbe, S.; Deguchi, M.; Hannya, Y. (2007). Basic research for a new airbag system for motorcycle, Proceedings of the $20^{\text {th }}$ International Technical Conference on the Enhanced Safety of Vehicles, Paper No. 07-0095, 9 pages

[11] Nakatani, T.; Sakurai, M.; Chawla, A.; Mukherjee, S. (2001). A methodology for motorcyclevehicle crash simulation - development of motorcycle computer simulation model, JARI Research Journal, Vol. 23, No. 10, 28-35

[12] Chawla, A.; Mukherjee, S.; Mohan, D.; Bose, D.; Rawat, P.; Sakurai, M.; Nakatani, T. (2003). FE simulations of motorcycle-car frontal crashes, validation and observations, Proceedings of the $18^{\text {th }}$ International Technical Conference on the Enhanced Safety of Vehicles, 40-57

[13] Chawla, A.; Mukherjee, S. (2007). Motorcycle safety device investigation: A case study on airbags, Sadhana, Vol. 32, No. 4, 427-443, doi:10.1007/s12046-007-0033-7

[14] Deguchi, M. (2005). Simulation of motorcycle-car collision, Proceedings of the $19^{\text {th }}$ International Technical Conference on the Enhanced Safety of Vehicles, Paper No. 05-0041, 7 pages

[15] Namiki, H.; Nakamura, T.; Iijima, S. (2003). A computer simulation for motorcycle rider-motion in collision, SAE/JSAE Small Engine Technology Conference \& Exhibition, SAE Paper No. 2003-32-0044, doi:10.4271/2003-32-0044

[16] Namiki, H.; Nakamura, T.; Iijima, S. (2005). A computer simulation for motorcycle rider injury evaluation in collision, Proceedings of the $19^{\text {th }}$ International Technical Conference on the Enhanced Safety of Vehicles, Paper No. 05-0309, 10 pages

[17] Bhosale, P. V. (2013). Exploratory study on the suitability of an airbag for an Indian motorcycle using finite element computer simulations of rigid wall barrier tests, Proceedings of the $23^{\text {rd }}$ International Technical Conference on the Enhanced Safety of Vehicles (ESV), Paper No. 130195, 16 pages

[18] Tan, K. S.; Wong, S. V.; Megat Ahmad, M. M. H. (2015). Computational simulation of frontal impact of motorcycle telescopic fork, International Journal of Crahsworthiness, Vol. 21, No. 2, 161-172, doi: $10.1080 / 13588265.2015 .1117770$

[19] Livermore Software Technology Corporation (2007). LS-DYNA Keyword User's Manual, Version 9.71, Livermore

[20] MODENAS. KRISS I, from: http://www.modenas.com.my/v2motorcycle.asp? id=2, accessed on 13-01-2015 
[21] Tan, C. L.; Tan, K. S.; Lim, Y. T.; Wong, S. V. (2008). Experimental analysis on static and impact response of motor cycle front fork, Proceedings of International Crashworthiness Conference (ICRASH 2008), Paper No. 117

[22] Hughes, T. J. R.; Liu, W. K. (1981). Nonlinear finite element analysis of shells: Part I. threedimensional shells, Computer Methods in Applied Mechanics and Engineering, Vol. 26, No. 3, 331-362, doi:10.1016/0045-7825(81)90121-3

[23] Hughes, T. J. R.; Liu, W. K. (1981). Nonlinear finite element analysis of shells: Part II. twodimensional shells, Computer Methods in Applied Mechanics and Engineering, Vol. 27, No. 2, 167-181, doi:10.1016/0045-7825(81)90148-1

[24] International Organization for Standardization (2011). ISO 15071: 2011 - Hexagon with Flange - Small Series - Product Grade A, Geneva, Switzerland

[25] Markiewicz, E.; Ducrocq, P.; Drazetic, P. (1998). An inverse approach to determine the constitutive model parameters from axial crushing of thin-walled square tubes, International Journal of Impact Engineering, Vol. 21, No. 6, 433-449, doi:10.1016/S0734-743X(98)00004-9

[26] Anghileri, M.; Chirwa, E. C.; Lanzi, L.; Mentuccia, F. (2005). An inverse approach to identify the constitutive model parameters for crashworthiness modelling of composite structures, Composite Structures, Vol. 68, No. 1, 65-74, doi:10.1016/j.compstruct.2004.03.001

[27] Japanese Standard Association (2010). JIS G3445:2010 - Carbon steel tubes for machine structure, Tokyo, Japan

[28] Sadlowska, H.; Kocanda, A. (2010). On the problem of material properties in numerical simulation of tube hydroforming, Archives of Civil and Mechanical Engineering, Vol. 10, No. 4, 77-83, doi:10.1016/S1644-9665(12)60033-8

[29] Parker, C. A.; Sikorsky, P. J.; Moosbrugger, C. (Eds.) (2002). Atlas of Stress-Strain Curves, $2^{\text {nd }}$ Edition, ASM International, Materials Park, Ohio 